\section{The effect of a peripheral stimulus on accommodation*}

\author{
ROBERT T. HENNESSY and H. W. LEIBOWITZ \\ The Pennsylvania State University, University Park, Pennsylvania 16802
}

Accommodation was measured by the laser scintillation technique while the $S$ viewed a stationary fixation spot through a series of apertures in a screen located at various distances. The magnitude of accommodation was a compromise between the distance of the fixation spot and the screen. Accommodation was affected significantly by the interaction of the distance of the screen with aperture sizes of 1 and $4 \mathrm{deg}$ and distance of the screen with its order of movement from near to far or far to near. The data are interpreted as implying the importance of the peripheral visual field and/or perceptual factors when conflicting cues to distance coexist in the visual field.

It is generally assumed that the eye will focus in a reflex manner to minimize the blur of the retinal image and that the receptors for the processes controlling this accommodation are localized in the central fovea (Fincham, 1951). There is some basis, however, to suspect that there are factors affecting accommodation in addition to the foveal stimulus. Disparity between the perceived and optical distance of a visual target is often cited as having an influence on accommodation (Landolt, 1886; Hofstetter, 1942; Ittelson \& Ames, 1950; Stark, 1968). The possible influence of the peripheral visual field has been disregarded probably because the poor quality of its image and low resolving power seem to exclude it as an important source of information. However, the phenomenon of instrument myopia, the unnecessary positive accommodation of the eye while looking through an optical instrument (Schober, 1954; Schober, Dehler, \& Kassel, 1970), suggests that the peripheral visual field may be involved. Even well-practiced microscopists, who would be expected to be minimally influenced by perceptual effects, may show 3 to 4 diopters of overaccommodation (Baker, 1966). In light of this, it is not unreasonable to assume that some aspect of a restricted visual field, characteristic of all optical instruments, may contribute to this unusual accommodation response.

The purpose of the present experiment is to test the hypothesis that psychological factors of a

*Sponsored by Grant MH 08061 from the National Institute of Mental Health, Public Health Service, and by a National Science Foundation traineeship to the senior author. Based in part on "Extra Foveal Influences on Accommodation," $R$. T. Hennessy's MS thesis, Pennsylvania State University, 1970. The authors are indebted to Professor Glenn A. Fry for his comments and encouragement. perceptual nature and/or influences of the peripheral visual field affect accommodation. The results of a number of experiments suggest that the vergence of light rays imaged on the fovea is sufficient to produce correct accommodative adjustments by the eye. It is the contention here that the perceptual factors discussed above are secondary influences which are normally in harmony with the physical stimulus and become significant only under certain viewing conditions. This occurs, for example, when perceptual cues to distance are in conflict with the physical cues provided by the vergence of the light rays emanating from the object or when viewing through an optical instrument. In order to analyze the influence of these secondary factors, it is necessary to create a situation in which strong perceptual and peripheral cues are pitted against a relatively weak foveal stimulus.

\section{METHOD}

The general method was to create a disparity between the physical and perceived distance of a fixation object in a viewing situation which included a homogeneous peripheral field and a limited central field (analogous to an optical instrument). These ends were accomplished by having the $S$ view a fixation spot through a circular aperture in a large white wall. The experimental room was well illuminated, and there were good cues to the distance of the wall. However, nothing was visible between the wall and the fixation spot, which was positioned on line, but behind, the center of the opening in the wall. It was hypothesized that, because of the paucity of cues to the distance of the fixation spot, it would tend to be localized in the plane of the wall in accordance with the equidistance tendency (Gogel, 1965b). The wall in this case is analogous to the field stop in an optical instrument. Thus, the perceived distance of the fixation spot and the peripheral stimulation would both be a function of the distance of the wall from the $S$, while the physical stimulus imaged on the fovea remained constant.

\section{Subjects}

Twenty-four undergraduate volunteers, 19 men and 5 women, aged 18 to 24 , served as Ss. All exhibited at least 20/25 near and far acuity with their left eyes.

\section{Apparatus}

The experimental room, the walls of which were hung with black draperies, was $12.2 \mathrm{~m}$ long, $2.7 \mathrm{~m}$ wide, and $2.13 \mathrm{~m}$ high. The $\mathrm{S}$, located in the center of the room, viewed monocularly a circular green fixation light located along the line of sight of his left eye $6 \mathrm{~m}$ from him. The fixation spot and the S's eyes were $1.29 \mathrm{~m}$ above the floor. The fixation spot, produced by rear-projecting a defocused spot image on a translucent white index card, subtended a visual angle of $.287 \mathrm{deg}$ of arc and had a luminance of $6.36 \mathrm{fL}$. A square fiberboard wall, $1.87 \mathrm{~m}$ on a side, painted white (reflectance $80 \%$ ), was located between $S$ and the fixation point and could be positioned from 4 to within $0.5 \mathrm{~m}$ from him. The central portion of the wall held one of a series of replaceable circular apertures. The center of each aperture was $1.29 \mathrm{~m}$ from the floor and located along the line of sight from S's left eye to the fixation spot. Two sets of apertures were used; the first subtended a visual angle of 1 deg and the second a visual angle of $4 \mathrm{deg}$ for all distances of the wall from the $S$. Luminance of the wall was constant at $\mathbf{1 5 . 9 2} \mathrm{fL}$.

Accommodative changes were measured by a method utilizing the scintillation pattern from a gas laser (Knoll, 1966; Baldwin \& Stover, 1968; Hennessy \& Leibowitz, 1970). When the diverged beam of a laser is reflectea rrom a surface, a peppery granularity is noted. If the $\mathrm{S}$ moves his head, the granularity will appear to move either with or against the head motion. The direction of the reported motion is directly related to the focal state of the eye. Movement of the laser pattern can be imparted conveniently by projecting the diverged laser beam on a slowly moving target drum. By placing a series of lenses in the optical path between S's eye and the laser target and noting the point of reversal of the motion, accommodation in force can be determined.

In the present experiment, the diverged laser beam was reflected from a cylinder, $10.2 \mathrm{~cm}$ in diam and $7.6 \mathrm{~cm}$ long, painted gold and rotated about a horizontal axis at $.1 \mathrm{rpm}$. The drum was located $1.34 \mathrm{~m}$ to the left of the $\mathrm{S}$ behind a black wall which 
Change in Accommodation (Relative to Wall at $4 \mathrm{~m}$ ) for Two Aperture Sizes and Three Distances of Wall

\begin{tabular}{ccccc}
\hline & & \multicolumn{3}{c}{ Distance of Wall } \\
\cline { 3 - 5 } Aperture Size & & $2 \mathrm{~m}$ & $1 \mathrm{~m}$ & $0.5 \mathrm{~m}$ \\
\hline \multirow{2}{*}{$4 \mathrm{Deg}$} & $\mathrm{M}$ & .11 & .26 & .50 \\
& $\sigma$ & .17 & .17 & .20 \\
$1 \mathrm{Deg}$ & $\mathrm{M}$ & .11 & .30 & .79 \\
& $\sigma$ & .10 & .28 & .48 \\
\hline
\end{tabular}

Change in Accommodation (Relative to Wall at $4 \mathrm{~m}$ ) for Two Orders of Presentation of Wall

\begin{tabular}{llccc}
\multirow{2}{*}{$\begin{array}{c}\text { Order of } \\
\text { Presentation }\end{array}$} & & \multicolumn{3}{c}{ Distance of Wall } \\
\cline { 3 - 5 } & & $2 \mathrm{~m}$ & $1 \mathrm{~m}$ & $0.5 \mathrm{~m}$ \\
\hline \multirow{2}{*}{ Near to Far } & $M$ & .07 & .31 & .78 \\
& $\sigma$ & .08 & .26 & .41 \\
Far to Near & $\mathrm{M}$ & .14 & .26 & .52 \\
\hline
\end{tabular}

occluded all but the central $4.2 \mathrm{deg}$ of the drum. The laser light reflected from the target drum, luminance $10.04 \mathrm{fL}$, was introduced into S's visual field by means of a beam-splitting prism, $20 \mathrm{~mm}$ on a side (transmission/reflectance $40 \%$ ), located in front of his left eye. A shutter allowed the $E$ to present the laser scintillation pattern for durations of 1 sec. A lens holder was located on the left side of the prism, such that the total length of the optical path between the cornea of S's eye to a lens in the holder was about $24 \mathrm{~mm}$. Lenses from a standard optometrist's trial set were employed.

\section{Procedure}

After screening with a Titmus Industrial-Professional Vision Tester, Ss were informed they were participating in a visual perception experiment. Instructions were read which explained their task and also served to familiarize them with the apparent movement of the laser scintillation pattern. The $\mathbf{S}$ was told to keep his head as motionless as possible in the chinrest, fixate the green spot of light, and report the direction of movement of the granularity when the laser light appeared. They were warned to continue to look at the green fixation target, even when the laser scintillation pattern appeared (in order to prevent possible shifts in accommodation resulting from an effort to look at the scintillation pattern). Between test series, Ss were encouraged to close their eyes, relax, or look around in order to avoid fatigue from prolonged staring at the fixation target.

After S's head was placed in the chinrest and the moveable wall was positioned, the $E$ turned on the fixation spot. The $\mathbf{S}$ was then asked to indicate in feet or inches, or both, the distance to the wall from his eyes and to indicate how far the green spot appeared to be from him. The $E$ would then say, "Ready," and flash the laser light a few seconds later. For the first test, no lens was placed in the holder. Depending on whether S's first response indicated under- or overaccommodation, a lens of plus or minus 1 diopter in the corrective direction was placed in the holder and the test sequence repeated. A bracketing technique was used, i.e., the strength of the lens used was usually about one-half more or less than the previous lens. The range of accommodation was considered to be established when adding or subtracting $1 / 4$ or $1 / 8$ diopter caused a reversal of movement to be reported. The final value was computed as the mean strength of the two lenses delimiting the range where a reversal was reported. It should be noted that this method reflects relative accommodation changes with great accuracy, but because of axial chromatic aberration a correction factor must be added for absolute values.

Two groups of. 12 Ss each viewed the fixation spot through apertures which subtended a constant visual angle of either 1 or $4 \mathrm{deg}$, respectively, for all distances of the wall. Order of movement of the wall from the far to the near position, or the reverse, was counterbalanced for both groups of Ss. The wall was positioned at successive distances of $4.0,2.0,1.0$, or $.5 \mathrm{~m}$.

\section{RESULTS}

The accommodative values are computed in terms of relative changes in accommodation for each distance of the wall, using the 4-m distance as a base value for each $S$. The data are presented in Tables 1 and 2 and plotted in Figs. 1 and 2 as relative change in accommodation as a function of the distance to the wall in meters.

If accommodation were determined solely by the foveal physical stimulus, no change would be evident and the functions in the figures would be horizontal. At the other theoretical extreme, if accommodation were determined only by the distance to the wall, the data would fall along the sloping dashed lines in the figures. It will be noted that all the data fall between these two theoretical extremes, reflecting the influence of both the foveal stimulus as well as the nonphysical factors introduced in this study. A repeated-measures analysis of variance summarized in Table 3 indicates that distance of the wall, order of presentation, and interaction between distance and aperture size and distance and order of presentation are all significant. While the effect of aperture size was not significant overall, a studentized range statistic

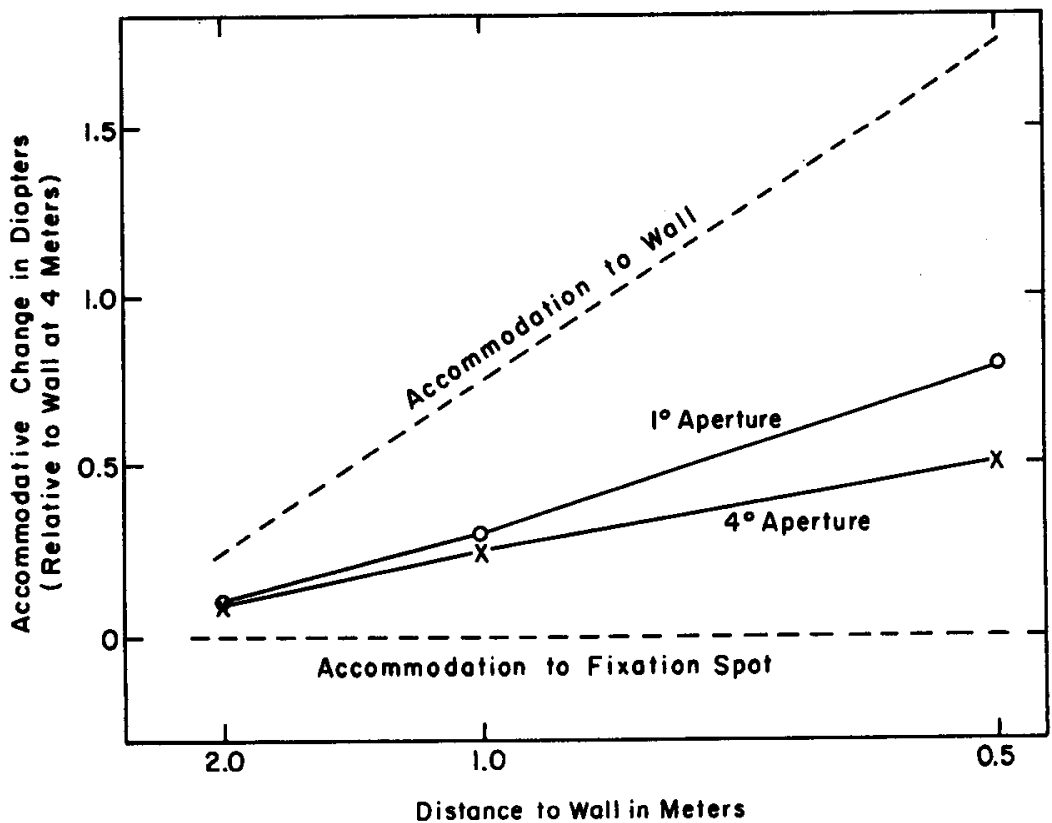

Fig. 1. Accommodative change in diopters as a function of distance to the wall in meters for a 1 -deg and for a $4-\mathrm{deg}$ aperture. 


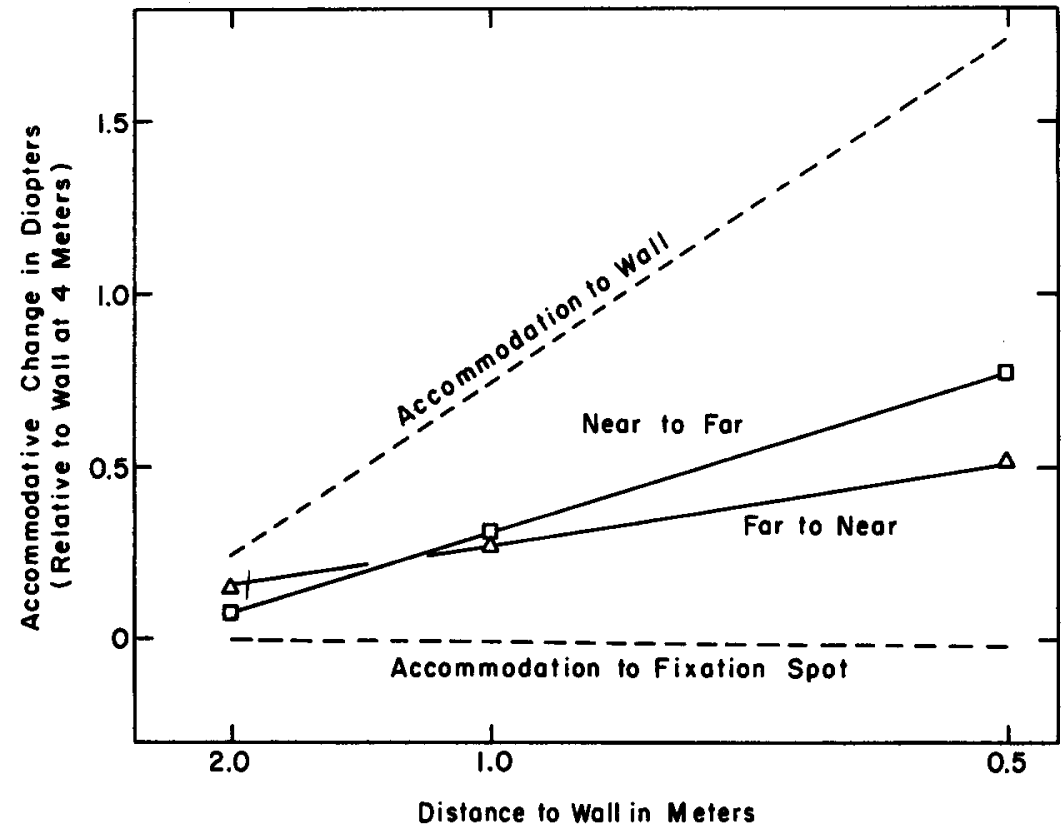

Fig. 2. Accommodative change in diopters as a function of distance to the wall in meters for the two orders of presentation of the wall.

$(\mathrm{q}=3.39, \mathrm{p}<.05)$ indicates a significant difference between aperture sizes when the wall was $.5 \mathrm{~m}$ from $\mathrm{S}$.

Combined median reported distances to the white wall and green fixation spot are shown in Table 4. Median reported distances did not differ significantly for aperture size or near-far conditions. These data are shown simply to indicate that the Ss did, in fact, perceive the green fixation spot to be perceptually nearer as the wall moved nearer and they have not been scaled to adjust for individual differences in reported distance.

In order to determine if the use of a defocused fixation spot contributed to the found changes in accommodation, data were obtained using a vertical cross as the fixation target. The arms of the cross were $.287 \mathrm{deg}$ long and $.45 \mathrm{~min}$ wide. The luminance was the same as the defocused spot. The Ss were five males from the same population as the main experiment.

Measurements of accommodation

were taken with the 4-deg aperture in the wall positioned successively at 4 , 1 , and $.5 \mathrm{~m}$ for three $\mathrm{Ss}$ and the reverse order for two. The defocused spot and the cross were used alternately as the fixation target at each position of the wall. The data are summarized in Table 5. A repeated-measures analysis of variance indicated no significant difference between the two types of fixation targets $(F=6.09$, df $=1 / 4, p>.05)$. As expected, there was a significant difference in the accommodative change for the two positions of the wall $(F=346.8, d f=1 / 4, p<.01)$.

\section{DISCUSSION}

It is clear from the data that the $\mathrm{Ss}$ accommodated as the wall moved toward them in spite of the fact that the distance of the central foveal stimulus was constant throughout the experiment. Either the perceived distance of the fixation spot or the large peripheral visual field stimulus

Table 3

Analysis of Variance Summary for Accommodation Data

\begin{tabular}{lccrr}
\multicolumn{1}{c}{ Source } & $\begin{array}{c}\text { Sum of } \\
\text { Squares }\end{array}$ & $\begin{array}{c}\text { Mean } \\
\text { Squares }\end{array}$ & df & F \\
\hline Between Ss & & & & \\
Aperture Size & .219 & .219 & 1 & 2.07 \\
Order of Movement & .119 & .119 & 1 & 1.12 \\
Aperture Size by Order & .183 & .183 & 1 & 1.17 \\
Error & 2.11 & .105 & 20 & \\
Within Ss & & & & \\
Distance of Wall & $\mathbf{3 . 6 0}$ & 1.80 & 2 & $42.03^{* *}$ \\
Distance by Aperture & .283 & .141 & 2 & $3.31 *$ \\
Distance by Order & .338 & .170 & 2 & $3.85 *$ \\
Distance by Aperture by Order & .049 & .024 & 2 & .568 \\
Error & $\mathbf{1 . 7 1}$ & .043 & 40 & \\
\hline
\end{tabular}

$* * p<.01, * p<.05$
Table 4

Median Reported Distance to Moveable Wall and Fixation Spot in Feet

\begin{tabular}{lrr}
\hline Distance of Wall & Wall & Spot \\
\hline $4 \mathrm{~m}(13.07 \mathrm{ft})$ & 15.0 & 16.0 \\
$2 \mathrm{~m}(6.53 \mathrm{ft})$ & 6.0 & 8.5 \\
$1 \mathrm{~m}(3.27 \mathrm{ft})$ & 3.0 & 6.0 \\
$.5 \mathrm{~m}(1.69 \mathrm{ft})$ & 1.7 & 3.5 \\
\hline
\end{tabular}

induced significant changes in accommodation contrary to the tacitly accepted assumption that the distance of the foveally fixated stimulus is the primary determinant of the focus of the eye.

It is generally recognized that several physical factors may contribute to accommodation. For example, Fincham (1951) has shown that although monochromatic light produces a temporary debility in the accommodative process, Ss can soon learn to make correct focal responses. This indicates that the loss of one factor can be compensated for by increased dependency on some other aspect of the stimulus situation. In the natural environment, all the possible influences usually act harmoniously and provide similar information about the correct accommodative setting to a specific point. If many objects are present in the visual field and are located at different distances, it is assumed that only the fixated object would determine accommodation. The present experiment demonstrates, however, that the visual context surrounding a somewhat degraded fixation object can importantly affect the focal state of the eye. This influence may arise out of perceptual cues, such as those included by Gogel's adjacency principle and equidistance tendency (Gogel, 1965a, 1965 b), or may be due to the attempt of the eye to attend to some significant object located in the peripheral field. Thus, in settings where the fixation object is impoverished, such as a small distant indistinct aircraft viewed through one of the narrow cabin windows of an airplane cockpit, objects in the peripheral field may exert a significant influence on accommodation. From the present study it is not possible to differentiate between factors arising

Table 5

Changes in Accommodation for a Defocused Spot and a Clearly Focused Cross (Relative to Wall at $4 \mathrm{~m}$ )

\begin{tabular}{cccc}
\hline & & \multicolumn{2}{c}{ Distance of Wall } \\
\cline { 3 - 4 } & & $1 \mathrm{~m}$ & $0.5 \mathrm{~m}$ \\
\hline Defocused & $\mathrm{M}$ & .28 & .71 \\
Spot & $\sigma$ & .20 & .42 \\
Cross & $\mathrm{M}$ & .30 & .82 \\
& $\sigma$ & .17 & .53 \\
\hline
\end{tabular}


from perceptual cues and those which may be a function of the peripheral field.

There is one aspect of the data which implies at least some involvement of perceptual factors. The data indicate that the order of movement of the wall either toward or away from the $S$ interacted significantly with distance of the wall. This implies that S's initial experience with the viewing situation affects accommodation. Since the Ss who first saw the wall in the near position did not know the length of the experimental room, they may have assumed that the fixation spot was initially much closer than did the group which first saw the wall at the far position and, consequently, may have been influenced by their awareness of the possible extreme distance of the fixation spot. This interaction of initial wall position and distance cannot be explained in terms of the peripheral field stimulus, since it was identical for both conditions.

It should be noted that the data of the present experiment suggest that neither the fixation spot nor the movable wall entirely determined accommodation, but rather that some sort of compromise between competing influences was reached. This compromise is consistent with the interpretation that the foveal stimulus is the primary determinant of accommodative change, but under certain circumstances, such as those which obtained in the present experiment, secondary influences can be of significance.

\section{REFERENCES}

BAKER, J. R. Experiments on the function of the eye in light microscopy. Journal of the Royal Microscopical Society, 1966 , 85, 231-254.

BALDWIN, W. R., \& STOVER, W. B. Observation of laser standing wave patterns to determine refractive status. American Journal of Optometry, 1968. 45, 143-150.

FINCHAM, E. F. The accommodation reflex and its stimulus. British Journal of Ophthalmology, 1951, 35, 381-393.

GOGEL, W. C. Size cues and the adiacency principle. Journal of Experimental Psycholozy, 1965a, 70, 289-293.

GOGEL, W. C. Equidistance tendency and its consequence. Psychological Bulletin, $1965 \mathrm{~b}, 64,153-163$.

HENNESSY, R. T:, \& LEIBOWITZ, H. W. Subjective measurement of accommodation with laser light. Journal of the Optical Society of America, 1970, 60, 1700-1701.

HOFSTETTER, H. W. The proximal factor in accommodation and convergence. American Journal of Optometry, 1942, 19, 66-76.

ITTELSON, W. H., \& AMES, A. Accommodation, convergence and their relation to apparent distance. Journal of Psychology, 1950, 30, 43-62.

KNOLL, H. A. Measuring ammetropia with a gas laser: A preliminary report. American Journal of Optometry, 1966, 43, 415-418.

LANDOLT, E. The refraction and accommodation of the eye. Philadelphia: Lippincott, 1886.

S C H O B E R, H. Ü b e $r$ di e Akkomodationruhelage. Optik, 1954, 11, 282-290.

SCHOBER, H. A. W., DEHLER, H., \& KASSEL, $R$. Accommodation during observations with optical instruments. Journal of the Optical Society of America, 1970, 60, 103-107.

STAR K, L. Neurological control systems. New York: Plenum Press, 1968.

(Accepted for publication January 10, 1971.) 